\title{
Small Scale Magnetic Field Evolution In The First Objects Formed In The Universe
}

\author{
Alejandra Kandus, Reuven Opher, \\ Departamento de Astronomía, IAG-USP, Rua do Matão 1226, Cidade Universitária, CEP: 05508-900, São Paulo, SP, Brazil \\ and Saulo M. R. Barros \\ Departamento de Matemática Aplicada, IME-USP, Rua do Matão 1010, Cidade Universitária, CEP: 05508-900, São Paulo, SP, Brazil
}

Received on 2 February, 2004; revised version on 19 May, 2004

\begin{abstract}
Large scale magnetic fields in galaxies are thought to be generated, by a mean field dynamo. In order to have generated the fields observed, the dynamo would have had to have operated for a sufficiently long period of time. However, magnetic fields of similar intensities to the one in our galaxy, are observed in high redshift galaxies, where a mean field dynamo would not have had time to produce the observed fields. MHD turbulence produces small scale magnetic fields at a faster rate than it does mean fields, which can diffuse toward larger scales. If the turbulence is helical, magnetic fields generated at small scales can become correlated over large scales. We study the evolution of magnetic field correlations in the first objects formed in the universe, due to the action of a turbulent, helical, stochastic dynamo, for redshifts $5 \leq z \leq 10$. Ambipolar diffusion can play a significant role in this process due to the low level of ionization of the gas in the first objects. We show that for reasonable values of the parameters that characterize the turbulent plasma in the time interval considered, fields can grow to high intensities $(\sim \mu \mathrm{G})$, with large coherence lengths $(\sim 2-6 \mathrm{kpc})$.
\end{abstract}

\section{Introduction}

The physical processes proposed to explain the origin and evolution of the magnetic fields detected in galaxies and clusters of galaxies, can be divided into two main classes: 1) cosmological mechanisms and 2) local astrophysical processes [1,2]. Until now, neither of them has provided a satisfactory explanation for the generation of the magnetic fields observed.

In order to explain the fields observed in our galaxy and in small redshift galaxies, a mean field dynamo is commonly invoked [3]. The dynamo would have had to have operated for a time on the order of the age of the universe to have attained the observed intensities. However, the presence of equally intense and coherent fields in high redshift galaxies $[4,5]$, where the mean field dynamo would not have had enough time to amplify the field to the observed values, casts doubt on the mean field dynamo paradigm as the preferred generation mechanism.

The incidence of star formation regions of the highest intensity increases monotonically with redshift [6]. Therefore the rate of occurrence of supernovae was also higher in the past than at present. Supernovae shocks disturb the plasma in which they are immersed, producing turbulent motions of the gas. If the occurence of supernovae was much higher in the past than at the present, the plasma of the first formed objects must have been more turbulent than observed galactic plasmas at low redshifts. These first stars that formed in the universe began to reionize the gas. Therefore the turbulence is MHD turbulence, with a degree of ionization far from complete or homogeneous.

It is known that MHD turbulence generates stochastic magnetic fields (magnetic noise) at a faster rate than mean fields. If the turbulence is strongly non-helical, the fields induced are confined to small scales [7, 8]. However if it is helical, magnetic field correlations over large scales occurs $[9,8]$.

In this study, we explore the hypothesis that the magnetic fields observed in high redshift galaxies are created by small scale, stochastic, turbulent helical dynamos, rather than mean field dynamos. Following Ref. [8], we take into account the backreaction of the growing magnetic fields on the turbulent plasma, in the form of ambipolar diffusion. The corresponding equations for the stochastic dynamo are therefore nonlinear in the magnetic field, producing a scale of coherence larger than those in linear theory.

\section{Our Model}

We study a gas cloud that is assumed to have collapsed at a high redshift, $z>10$. At $z \sim 10$, the cloud would have had a low magnetization level and a high level of turbulence. Thus, it would have been similar to the turbulent, low ionization molecular clouds observed in our galaxy, albeit with a much smaller initial magnetic field and a higher level of turbulence.

In all galaxies, the supernova rate is a direct measure of the cosmic ray intensity [10]. Thus, we can infer that cosmic rays were already present in considerable intensities in high 
redshift galaxies, modifying the plasma turbulence as they do in our galaxy. We take into account phenomenologically, the effect of cosmic rays, supernova shocks, and powerful stellar winds from massive stars by varying the turbulent parameters over a broad range.

\subsection{Magnetic field evolution equations}

The evolution equation for the magnetic field is given by the induction equation

$$
\partial \mathbf{B} / \partial t=\nabla \times(\mathbf{v} \times \mathbf{B}-\eta \nabla \times \mathbf{B}),
$$

where $\mathbf{B}$ is the magnetic field, $\mathbf{v}$ the velocity of the fluid and $\eta$ is the Ohmic resistivity. $\mathbf{v}\left(=\mathbf{v}_{T}+\mathbf{v}_{D}\right)$ is the sum of an external stochastic field component $\mathbf{v}_{T}$, and an ambipolar drift component $\mathbf{v}_{D}$, which describes the non-linear backreaction of the Lorentz force. This back-reaction is due to the force that the ionized gas exerts on the neutral gas through collisions of the ions with the neutral atoms.

We take B to be a homogeneous, isotropic, Gaussian random field with a negligible mean value. Therefore, we take the equal time, two point correlation of the magnetic field as $\left\langle B^{i}(\mathbf{x}, t) B^{j}(\mathbf{y}, t)\right\rangle=M^{i j}(r, t)$, where

$$
M^{i j}=M_{N}\left[\delta^{i j}-\left(\frac{r^{i} r^{j}}{r^{2}}\right)\right]+M_{L}\left(\frac{r^{i} r^{j}}{r^{2}}\right)+H \epsilon_{i j k} r^{k}
$$

and $r=|\mathbf{x}-\mathbf{y}|$. The symbol \langle\rangle denotes a double ensemble average over both the stochastic velocity and stochastic B fields. $M_{L}(r, t)$ and $M_{N}(r, t)$ are the longitudinal and transverse correlation functions respectively, of the magnetic field. $H(r, t)$ is the helical term of the correlations. The induction equation can be converted into evolution equations for $M_{L}$ and $H$ [8]:

$$
\begin{aligned}
\frac{\partial M_{L}}{\partial t}(r, t) & =\frac{2}{r^{4}} \frac{\partial}{\partial r}\left(r^{4} \kappa_{N}(r, t) \frac{\partial M_{L}(r, t)}{\partial r}\right) \\
& +G M_{L}(r, t)+4 \alpha_{N} H(r, t), \\
\frac{\partial H}{\partial t}(r, t) & =\frac{1}{r^{4}} \frac{\partial}{\partial r}\left[r ^ { 4 } \frac { \partial } { \partial r } \left[2 \kappa_{N}(r, t) H(r, t)\right.\right. \\
& \left.\left.-\alpha_{N}(r, t) M_{L}(r, t)\right]\right],
\end{aligned}
$$

where

$$
\begin{gathered}
\kappa_{N}(r, t)=\eta+T_{L L}(0)-T_{L L}(r)+2 a M_{L}(0, t), \\
\alpha_{N}(r, t)=2 C(0)-2 C(r)-4 a H(0, t),
\end{gathered}
$$

and

$$
G(r)=-4\left\{\frac{d}{d r}\left[\frac{T_{N N}(r)}{r}\right]+\frac{1}{r^{2}} \frac{d}{d r}\left[r T_{L L}(r)\right]\right\} .
$$

The velocity $\mathbf{v}_{T}$ is assumed to be an isotropic, homogeneous, Gaussian random field, with a zero mean value and a delta function correlation in time (Markovian approximation). Its two point correlation function is formlly identical to the one for the magnetic field (3), with $M_{L}$ replaced by $T_{L L}, M_{N}$ by $T_{N N}$ and $H$ by $C$. The velocity $\mathbf{v}_{D}=$ $a[(\nabla \times \mathbf{B}) \times \mathbf{B}]$, where $a=\tau / 4 \pi \rho_{i}, \tau$ is the characteristic response time, and $\rho_{i}$ is the ion density. $\eta$ is the microscopic diffusion coefficient. $T_{L L}(0)-T_{L L}(r)$ are the scale dependent turbulent diffusion coefficients. $2 a M_{L}(0, t)$ is the correction due to ambipolar diffusion. $2 C(0)-2 C(r)$ is the scale-dependent $\alpha$ effect (responsible for inducing magnetic fields correlated on scales larger than $\left.L_{c}\right)$. $4 a H(0, t)$ is the nonlinear decrement of the $\alpha$ effect due to ambipolar diffusion. Finally, $G(r)$ is a term which allows for the rapid generation of magnetic fluctuations due to velocity shear and the existence of the small-scale dynamo.

We are interested in the evolution of $M_{L}$, since this function provides information about the coherence of the induced large scale field. A positive value of this function over a given length indicates that the magnetic field is coherent in this region. Therefore this length will be taken as the coherence scale of the induced field. The maximum scale of coherence attained in each case can be estimated as the region about $r=0$, within which $M_{L}$ is positive. $M_{L}$ is the tensor product of parallel field vectors, evaluated at two points separated by a distance $r$. We can estimate the induced magnetic field intensity at all points where $M_{L}>0$ as $B \sim M_{L}(r) / M_{L}^{1 / 2}(0)$

\subsection{Characterizing the high redshift plasma}

We considered a cloud at $z \sim 10$ and followed the evolution of the magnetic correlations until $z \sim 5\left(\sim 10^{9}\right.$ years $)$. The value taken for the cut-off scale of the turbulence, $l_{c} \sim 1 \mathrm{AU}$, is similar to that for present objects [2]. Assuming $L_{c} \gg l_{c}$, we studied the range of values $10 \mathrm{pc} \lesssim$ $\mathrm{L}_{\mathrm{c}} \lesssim 100$ pc. We assumed that the height $h$ of the turbulent eddies of the high redshift object is of the same order of magnitude as $L_{c}$. In order to estimate the correlation velocity $V_{c}$ on the scale $L_{c}$, we used the expression $V_{c}^{2}\left(V_{c} / L_{c}\right) \sim \varepsilon$, where $\varepsilon$ is the turbulent energy dissipated per unit mass per unit time. This expression assumes that the energy is dissipated on the order of a single rotation of the eddies of size $L_{c}$ at the angular frequency $\Omega \sim V_{c} / L_{c}$. We then have $V_{c} \sim\left(\varepsilon L_{c}\right)^{1 / 3}$. Supernova explosions are a major contributor to the galactic turbulent energy. The energy associated with a supernova remnant in our galaxy is about $3 \times 10^{50} \mathrm{erg}$, with about one third transformed into kinetic energy of the ambient gas. Larger values for the supernova remnant energy and the mass of the gas involved in the explosions, will produce higher turbulent velocities. We assumed that at redshifts 5-10, $f$ explosions occurred every 5 years and that the mass of the gas involved was $10^{10} M_{\odot}$ [2]. As noted above, the star formation and supernova rates were very high in the past. The indicated star formation rate from observations increased by a factor of $\sim 50$, in going from $z \sim 0$ to $z \sim 8$ (see e.g., fig. 4 in Lanzetta et al. [6]). The expected values for $f$ are then $1<f \lesssim 10$. A value of $f \sim 0.1$ corresponds to the present supernova rate in our galaxy. We, thus, have $\varepsilon \simeq 0.3 \times f \mathrm{~cm}^{2} \mathrm{~s}^{-3}$. For the considered values of $L_{c}$, the expected range of values for $V_{c}$ is $9.59 \mathrm{~km} \mathrm{~s}^{-1} \lesssim V_{c} \lesssim 96.5 \mathrm{~km} \mathrm{~s}^{-1}$. These values are 3 - 10 times larger than those in our galaxy [2]. Assuming that the largest velocity corresponds to the largest eddy, we have $\Omega \sim 10^{-13} \mathrm{~s}^{-1}$. We estimated that the baryon density is $\rho_{n}(z)=\rho_{n}(0)(1+z)^{3} b$, where $\rho_{n}(0)$ is the present 
baryon density and $b$ is a compression factor, which can be much greater than $\sim 200$ (virial collapse). In our galaxy, the particle density is $\sim 1 \mathrm{~cm}^{-3}$ or $\rho_{n} \sim 10^{-24} \mathrm{~g} \mathrm{~cm}^{-3}$. The average baryon density in the universe today is $\sim$ $10^{-30} \mathrm{~g} \mathrm{~cm}^{-3}$. Thus, for our galaxy, the compression factor is $b \sim 10^{6}$. We assumed that the cloud that we are studying in the interval $5 \leq z \leq 10$, collapsed virially at a high redshift, creating a large $b$. Reasonable values for $b$ are, then, in the range $200 \leq b \leq 10^{7}$. Taking $\rho_{n}(0) \sim 0.05 \rho_{c}(0)$, where $\rho_{c}(0) \simeq 0.9 \times 10^{-29} \mathrm{~g} \mathrm{~cm}^{-3}$ is the present critical density (assuming a fiducial factor, $h \sim 0.7$, for the Hubble constant), we obtain $4 \times 10^{-26} \mathrm{~g} \mathrm{~cm}^{-3} \lesssim \rho_{\mathrm{n}}(z=$ $10) \lesssim 2.3 \times 10^{-21} \mathrm{~g} \mathrm{~cm}^{-3}$ for the baryon density in our high redshift cloud. We estimated the ion mass density as $\rho_{i} \sim g \rho_{n}$, with $0.001 \lesssim g \lesssim 1$, which gives an ion density in the range $4 \times 10^{-29} \mathrm{~g} \mathrm{~cm}^{-3} \lesssim \rho_{\mathrm{i}} \lesssim 2.3 \times 10^{-21} \mathrm{~g} \mathrm{~cm}^{-3}$. At $z \sim 10$, the cosmic microwave radiation temperature was $(1+z) T_{0} \sim 30 \mathrm{~K}$. For $5 \lesssim z \lesssim 10$, we considered plasma cloud temperatures in the interval $30 \mathrm{~K} \lesssim \mathrm{T} \lesssim 10^{3} \mathrm{~K}$. Using these values and estimating the thermal velocity of the ions as $v_{n}=\left(3 k_{B} T / m_{p}\right)^{1 / 2}$, we obtained $10^{4} \mathrm{~cm} \mathrm{~s}^{-1} \lesssim$ $v_{n} \lesssim 10^{5} \mathrm{~cm} \mathrm{~s}^{-1}$. Comparing these values with $V_{c}$, we see that we are dealing with mildly supersonic turbulence. Due to the the relatively low temperatures of the plasma, the ionneutral collision cross section is $\sigma_{i n} \simeq 10^{-15} \mathrm{~cm}^{2}$ [13]. The ion-neutral collision frequency is $\nu_{i n}=\sigma_{i n} n_{n} v_{t h}$, giving $10^{-16} s^{-1} \lesssim \nu_{i n} \lesssim 10^{-10} s^{-1}$. The electrical resistivity can be estimated as $\eta=\left(c^{2 / 4} \pi\right)\left(m_{e} \nu_{e n} / e^{2} n_{e}\right)$, where $n_{e}$ is the electron number density, $m_{e}$ the electron mass, and $\nu_{e n}=\left\langle\sigma_{e n} v_{e}\right\rangle n_{n}$ is the electron-neutral collision frequency. Taking $n_{e}=n_{i}$ (charge neutrality), $T_{e} \sim T_{i}$, and using $v_{e} \sim\left(3 k_{B} T_{e} / m_{e}\right)^{1 / 2}$, we obtain $\eta \sim 5 \times 10^{3} \mathrm{~cm}^{2} \mathrm{~s}^{-1}$, which is extremely small. The magnetic Reynolds number is $R_{m}=L_{c} V_{c} / \eta \sim 10^{23}-10^{24}$, which means that at high redshifts, plasma turbulence was the main mechanism for diffusion and dissipation. Thus the first term in equation (5) can be neglected. Since the ionneutral collision was the dominant interaction in the plasmas considered, we took the characteristic response time as $\tau \sim \nu_{i n}^{-1}$. The coefficient " $a$ " in the non-linear terms in equations (3) and (4) can then assume values in the interval $4.3 \times 10^{30} \mathrm{~g}^{-1} \mathrm{~cm}^{3} \mathrm{~s} \lesssim a \lesssim 2.5 \times 10^{44} \mathrm{~g}^{-1} \mathrm{~cm}^{3} \mathrm{~s}$.

\subsection{Characterizing the turbulence}

In studying turbulence, it is usually assumed that the fluid is incompressible $(\nabla \cdot \mathbf{v}=0)$. The functions $T_{N N}$ and $T_{L L}$ are, then, related in the way described by Subramanian [8]. For compressible fluids, $\left(\nabla \times \mathbf{v}_{T}=0\right)$ these functions are related by $T_{L L}=T_{N N}+r d T_{N N} / d r$ [11]. Adopting the above relation between $T_{N N}$ and $T_{L L}$, since astrophysical plasmas are compressible, the fluid flow correlation functions can be written as

$$
\begin{array}{rlrl}
2 C(r) & =\frac{\Omega L_{c}^{2}}{h}\left[1-\left(\frac{r}{L_{c}}\right)^{q}\right] & & 0<r<L_{c} \\
T_{N N}(r) & =A_{N}\left[1-\left(\frac{r}{L_{c}}\right)^{p}\right] & & l_{c}<r<L_{c} \\
T_{N N}(r) & =0 \quad r>L_{c} &
\end{array}
$$

with $A_{N}=V_{c} L_{c} / 3$ [12]. (In our study, $l_{c}$ is much smaller than the numerical resolution used. We therefore considered $\left.M_{L}(0)=M_{L}\left(l_{c}\right)\right)$.

\section{Results and conclusions}

The magnetic correlations that result from the evolution of the turbulent kinematical dynamo are found to be independent of the initial field correlations. The resulting intensity of the magnetic field is very sensitive to the value of both $V_{c}$ and the degree of ionization of the plasma, while the final correlation length $L_{M}$ of the magnetic field is found to be very sensitive to the values of $V_{c}$ and $\Omega$. Larger values of $p$ and/or of $q$ produce larger values of $M_{L}$.

To illustrate the dependence of varying the parameters, we investigated the following turbulent cases:

- $L_{c}=33 \mathrm{pc}, V_{c}=45 \mathrm{~km} \mathrm{~s}^{-1}$ and $\Omega=4.5 \times 10^{-13}$ $\mathrm{s}^{-1}$. We obtained an average magnetic field $B \sim$ $1.1 \times 10^{-6} \mathrm{G}$ and a final correlation length, $L_{M} \simeq 1.7$ kpc.

- $L_{c}=80 \mathrm{pc}, V_{c}=96 \mathrm{~km} \mathrm{~s}^{-1}$ and $\Omega=9.6 \times 10^{-13}$ $\mathrm{s}^{-1}$. We obtained an average magnetic field $B \sim$ $1.4 \times 10^{-6} \mathrm{G}$ and a final correlation length, $L_{M} \sim 5.4$ kpc.

In both cases we took $p=4 / 3, q=2, \eta \sim 10^{3} \mathrm{~cm}^{2}$ $\mathrm{s}^{-1}$, and $a \simeq 9.76 \times 10^{39} \mathrm{~cm}^{3} \mathrm{~s} \mathrm{~g}^{-1}$.

In Fig. 1 we plot the final $M_{L}$, for different values of $a$. We show the final $M_{L}$ for different values of $V_{c}$ in Fig.2. The growth of $M_{L}$ as a function of time for various initial conditions is shown in Fig. 3.

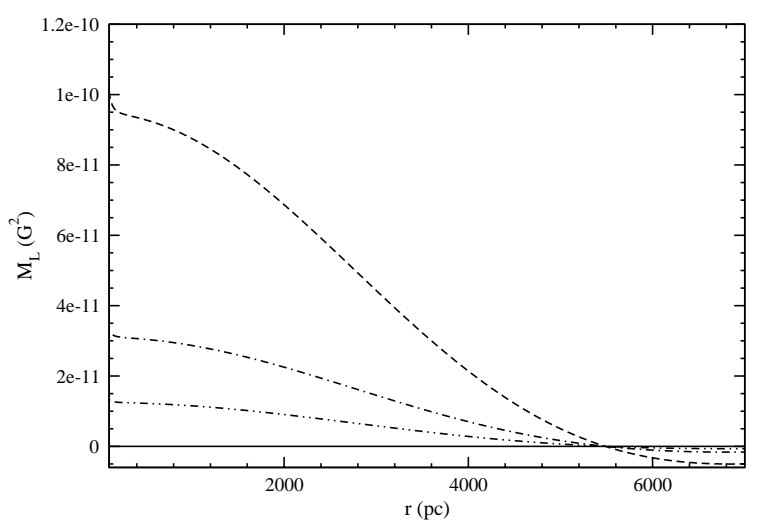

Figure 1. Final magnetic longitudinal correlations, $M_{L}\left(\mathrm{G}^{2}\right)$ as a function of $r(\mathrm{pc})$, for $q=2, p=1.333, L_{c}=83 \mathrm{pc}$, $\Omega=0.98 \times 10^{-13} \mathrm{~s}^{-1}$ and $V_{c}=98 \mathrm{~km} / \mathrm{s}$. Three different values for $a$ are shown, $a=3.2 \times 10^{39} \mathrm{~cm}^{3} \mathrm{~s} \mathrm{~g}^{-1}$ (dashed line), $9.7 \times 10^{39} \mathrm{~cm}^{3} \mathrm{~s} \mathrm{~g}^{-1}$ (dash-dotted line) and $2.43 \times 10^{40} \mathrm{~cm}^{3} \mathrm{~s}$ $\mathrm{g}^{-1}$ (dash-doubledotted line). We see that the smaller the value of $a$, the larger are the magnetic field intensities. The coherence scale remains practically unchanged.

Figure 3 shows the rapid saturation $\left(\sim 5 \times 10^{6}\right.$ years $)$ for small scales, $r \sim 112 \mathrm{pc}$. This is in agreement with the analysis of Kulsrud and Anderson [14]. Larger scales take a longer time to saturate. For the distance $r \sim 2000-4000$ pc, 


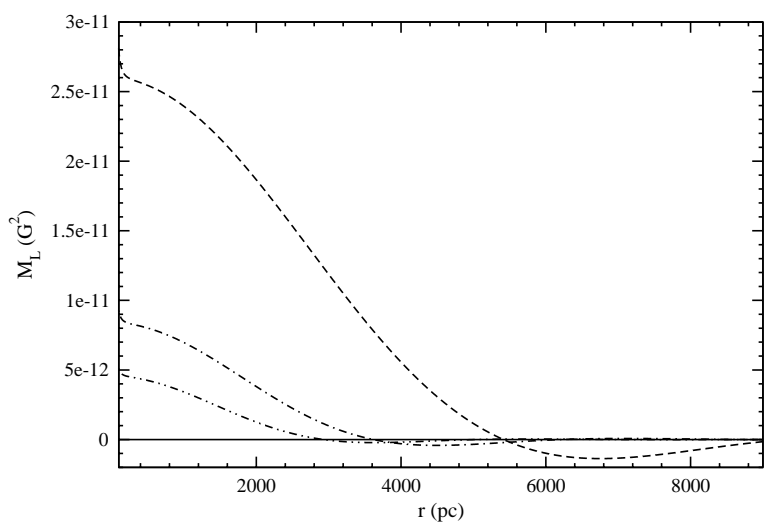

Figure 2. Final magnetic longitudinal correlations, $M_{L}\left(\mathrm{G}^{2}\right)$ as a function of $r$ (in pc), for $q=2, p=1.33, L_{c}=83 \mathrm{pc}$ and $a=3.2 \times 10^{39} \mathrm{~cm}^{3} \mathrm{~s} \mathrm{~g}^{-1}$. Three different values for $V_{c}$ are shown, $V_{c}=30 \mathrm{~km} \cdot \mathrm{s}^{-1}$ (dash-doubledotted line), $45 \mathrm{~km} \mathrm{~s}^{-1}$ (dash-dotted line) and $96 \mathrm{~km} \mathrm{~s}^{-1}$ (dashed line), with corresponding values of $\Omega=0.3 \times 10^{-13} \mathrm{~s}^{-1}, 0.45 \times 10^{-13} \mathrm{~s}^{-1}$ and $0.96 \times 10^{-13} \mathrm{~s}^{-1}$. We see that for larger values of $V_{c}$, larger field intensities and coherence scales are obtained.

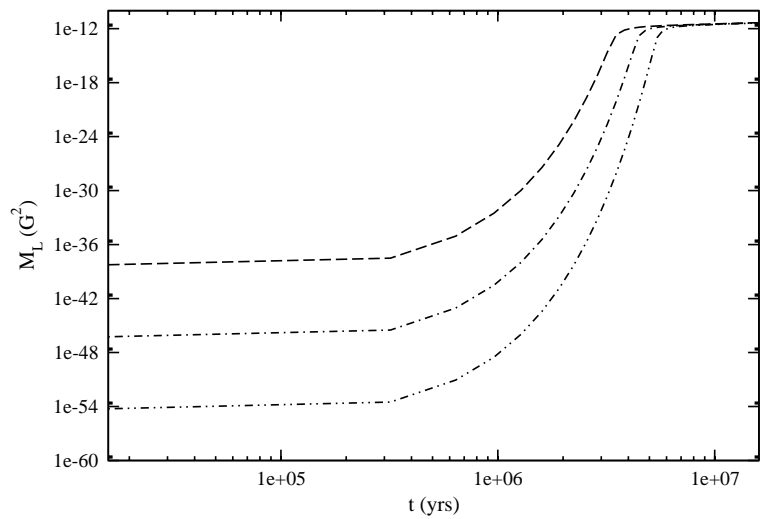

Figure 3. Magnetic longitudinal correlation, $M_{L}\left(\mathrm{G}^{2}\right)$ as a function of $t$ (yrs), for $r_{0}=112 \mathrm{pc}$ and for three different initial values, $M_{L}\left(r_{0}, t=0\right)=1.5 \times 10^{-38} \mathrm{G}^{2}$ (dashed line), $1.5 \times 10^{-47} \mathrm{G}^{2}$ (dash-dotted line) and $1.5 \times 10^{-55} \mathrm{G}^{2}$ (dash-doubledotted line). We used $p=1.11, q=2, L_{c}=81 \mathrm{pc}, \Omega=0.75 \times 10^{-13} \mathrm{~s}^{-1}$, $V_{c}=98 \mathrm{~km} \mathrm{~s}^{-1}, a=9.76 \times 10^{38} \mathrm{~cm}^{3} \mathrm{~s} \mathrm{~g}^{-1}$. We see that independent of the initial values of $M_{L}$, saturation occurs at a time $t \sim 5 \times 10^{6}$ years when $M_{L}^{1 / 2} \sim 10^{-6} \mathrm{G}$.

shown in Figs. 1 and 2, we require the entire time interval $5<z<10$ for longitudinal correlations to develope.

The age of the universe at $z=10$ is $4.7 \times 10^{8}$ years. A galaxy at this redshift has already been observed [15]. The star formation rate in this galaxy is observed to be extremely high. A high level of turbulence produced by supernovae and star formation can then be assumed to exist already at $z \sim 10$ and, thus, throughout the redshift interval $5<z<10$.

In summary, we found that for a reasonable set of turbulent, astrophysical parameters, magnetic fields on the or- der of $10^{-6} \mathrm{G}$, as are observed in high redshift objects are generated in less than about $10^{9}$ years (the time elapsed between $z \sim 10$ and $z \sim 5$ ). They are coherent on scales of $L_{M} \simeq 3.5-5.4 \mathrm{kpc}$.

Our model for the generation and evolution of magnetic correlations is relatively simple. Our results, which are preliminary due to the simple evolution equations used, suggest that the reionization process of the universe, involving the formation of the first stars, played an important role in determining the features of the magnetic fields detected in high redshift objects.

\section{Acknowledgments}

We thank George Morales for useful comments. We acknowledge E. Opher for careful proof reading of this manuscript. This work was partially supported by the Brazilian financing agency FAPESP (00/06770-2). A.K. acknowledges the FAPESP fellowship (01/07748-3). R. O. acknowledges partial support from the Brazilian financing agency $\mathrm{CNPq}$ (300414/82-0).

\section{References}

[1] D. Grasso and H. Rubinstein, Phys. Rept. 348, 163 (2001); L. M. Widrow, Rev. Mod. Phys. 74, 775 (2002)

[2] Ya. B. Zel'dovich, A. A. Ruzmaikin and D. D. Sokoloff, Magnetic Fields in Astrophysics, (Gordon and Breach, New York, 1983).

[3] K. H. Moffat, Magnetic Field Generation in Electrically Conducting Fluids, (Cambridge Univ. Press, Cambridge, 1978)

[4] C. L. Carrilli and R. B. Taylor, ARA\&A 40, 319 (2002).

[5] A. M. Wolfe, K. M. Lanzetta and A. L. Oren, 1 Astrophys. J. 388, 17 (1992).

[6] K. M. Lanzetta, N. Yahata, S. Pascarelle et al., Astrophys. J. 570, 492 (2002).

[7] A. P. Kazantzev, JETP 26, 1031 (1968).

[8] K. Subramanian, Phys. Rev. Lett. 83, 2957 (1999).

[9] S. I. Vainshtein and L. L. Kichatinov, J. Fluid Mech. 168, 73 (1986),

[10] H. J. Völk, U. Klein, and R. Wielebinski, A\&A 213, L12 (1989).

[11] A. S. Monin, and A. A. Yaglom, A. A. Statistical Fluid Mechanics, (MIT Press, Cambridge 1975).

[12] S. I. Vainshtein, JETP 56, 86 (1982).

[13] Naval Research Laboratory 2002, Plasma Formulary

[14] R.M. Kulsrud and S. W. Anderson, Astrophys. J. 396, 606 (1992).

[15] R. Pello, D. Schaerer, J. Richard, J. -F. Le Borgne and J. -P. Kneib, astro-ph/0403025. 